\title{
Deep Learning Framework for Detection of Hypoglycemic Episodes in Children with Type 1 Diabetes*
}

\author{
Phyo Phyo San, Sai Ho Ling, Senior Member, IEEE, and Hung T. Nguyen, Senior Member, IEEE
}

\begin{abstract}
Most Type 1 diabetes mellitus (T1DM) patients have hypoglycemia problem. Low blood glucose, also known as hypoglycemia, can be a dangerous and can result in unconsciousness, seizures and even death. In recent studies, heart rate (HR) and correct QT interval (QTc) of the electrocardiogram (ECG) signal are found as the most common physiological parameters to be effected from hypoglycemic reaction. In this paper, a state-of-the-art intelligent technology namely deep belief network (DBN) is developed as an intelligent diagnostics system to recognize the onset of hypoglycemia. The proposed DBN provides a superior classification performance with feature transformation on either processed or un-processed data. To illustrate the effectiveness of the proposed hypoglycemia detection system, 15 children with Type 1 diabetes were volunteered overnight. Comparing with several existing methodologies, the experimental results showed that the proposed DBN outperformed and achieved better classification performance.
\end{abstract}

\section{INTRODUCTION}

Hypoglycemia (low blood glucose) can be encountered in any Type 1 diabetes mellitus (T1DM) patients who take medicines for increasing levels of insulin in their body. Particularly, it is very dangerous during the sleep time as it reduces or obscures autonomic counter-regulatory responses. In [1], it is reported that at least half of severe hypoglycemia episodes occurring at night time. The blood glucose level is considered low (hypoglycemia) when it drops below $3.3 \mathrm{mmol} / \mathrm{L}$ [2], and patients are advised to take necessary treatment. When the initial symptoms of hypoglycemia occur [3], the patient can then recognize these in ensuing episodes.

Numerous non-invasive blood glucose monitoring systems are currently available in the market to measure blood glucose concentration. However, there are several drawbacks in terms of cost, functioning, reliability and obtrusiveness. Intensive research has been conducted to develop a hypoglycemia detector by using glucose sensors from detecting changes in the electroencephalogram (ECG) signals and skin conductance [4]. In 2006, a real-time monitoring systems (CGMS) were developed [5]. However, they were not developed as commercial devices due to lack of sensitivity and low efficiency in detecting unrecognized hypoglycemia.

This paper will make a significant contribution to knowledge in the modeling and design of a non-invasive hy-

\footnotetext{
*The authors would like to thank Dr. Nejhdeh Ghevondian, and Assoc. Prof. Timothy Jones for their contribution. This works was supported by a grant from Juvenile Diabetes Research Foundation.

P.P. San is with the Data Analytic Department, Institute for Infocomm Research, Singapore.e-mail: sanppei2r.a-star.edu.sg

S.H. Ling and H.T. Nguyen are with the Center for Health Technologies, Faculty of Engineering and IT, University of Technology Sydney, Ultimo, NSW, Australia.e-mail: Steve.Ling@uts.edu.au; Hung. Nguyenduts . edu . au
}

poglycemia monitor using physiological responses. During hypoglycemia, the most profound physiological changes are caused by activation of the sympathetic nervous system. Among them, the strongest responses are sweating and increased cardiac output [6]. Sweating is mediated through sympathetic cholinergic fibres, while the change in cardiac output is due to an increase in heart rate and increase in stroke volume. In [7], experimental hypoglycemia has been shown that prolongation of QT intervals and dispersion occur in both non-diabetic subjects and Type 1 and Type 2 diabetic patients.

With the use of the physiological parameters, hypoglycemia detection systems have been developed by blockbased neural network (BBNN) [8], fuzzy reasoning model (FRM) [9], wavelet neural network (WNN) [10], feedforward neural network (FFNN) [10] and multiple regression (MR). Though satisfactory results are found by the use of the advanced neural networks and fuzzy reasoning models, aiming for better accuracy is always essential for more accurate detection of hypoglycemia episodes.

In recent years, deep learning has been successfully applied in various domains, ranging from image classification [11], speech recognition to audio classification [12]. Besides the successful stories of image and speech recognition, these approaches have been introduced in physiological signals such as electromyogram (EMG), electrocardiogram (ECG) and electroencephalography (EEG) [13] and obtained better accuracy. These recent surges of DBN activities are largely spurred to use in hypoglycemia detection system in order to overcome the shortcomings encountered in standard neural network classifiers.

In this paper, a recent advanced DBN is introduced in order to detect the status of hypoglycemia episodes. The inputs in this strategy are features heart rate (HR) and QTc of QRS wave. With the use of DBN, high-level ECG features are preliminarily represented through the layer-wise training of deep hierarchical model and followed by standard $\mathrm{NN}$ for fine-tuning and classification purposes. The overall operation of the DBN-based hypoglycemia monitoring system in this study consists of three main components which are data collection and feature selection, high-level features representation using DBN and classification which are hypo or non-hypo states.

The organization of this paper is as follows: in Section II, a DBN and its training procedures by the use of constructive divergence (CD) algorithm is introduced. To show the effectiveness of our proposed methods, the results of early detection of nocturnal hypoglycaemia episodes in T1DM are 
discussed in Section III and a conclusion is drawn in Section IV.

\section{METHODS}

To recognize the status of hypoglycemia episodes in T1DM by use of physiological parameters of ECG signal (Fig. 1), a deep learning framework (Fig. 2) is developed. In Fig. 1, the ECG signal is composed of a $\mathrm{P}$ wave which represents atrial depolarization while a QRS complex and a T wave represents ventricular depolarization and rapid change of repolarization of ventricles.

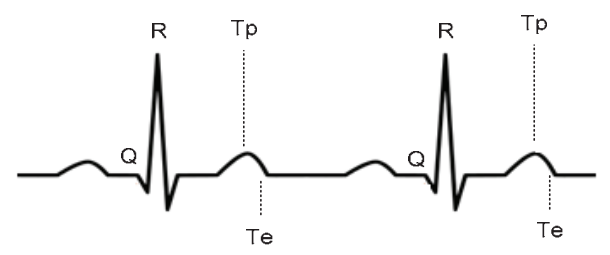

Fig. 1. ECG Signal

For patients with Type 1 and Type 2 diabetes, the possibility of hypoglycemia is mainly effected by prolongation of QT intervals (starting from the point of $\mathrm{Q}$ wave to at the end of $\mathrm{T}$ wave) and its correlation to heart rate carried out by Bazett's formula $Q T_{c}=Q T / R R$ [14]. Not only does $Q T_{c}$ interval prolongation have a significant impact on hypoglycemia, but an increase in heart rate (HR) may also influence the status of hypoglycemia [15]. Studies on the natural occurrence of hypoglycemia with an increase in heart rate, $(1.033 \pm 0.242$ vs. $1.082 \pm 0.298, P<0.06)$ and corrected QT intervals, $(1.031 \pm 0.086 v s .1 .060 \pm 0.084, P<0.01)$ have been successfully carried out in [16].

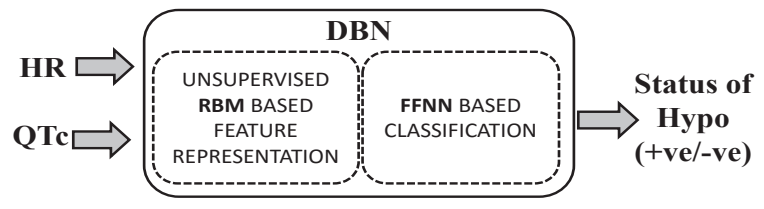

Fig. 2. The proposed DBN based EEG Classification System

With these changes in physiological parameters, a deep belief network (DBN) model is developed for early detection of hypoglycemic episodes. The DBN-based hypoglycemia monitoring system is developed with two inputs (heart rate (HR) and corrected QT interval $\left(Q T_{c}\right)$ ), and one output system (hypoglycemia status) as presented in Fig. 2 in which the restricted boltzmann machines (RBM) (Sec. II-.2) and feed-forward neural network (FFNN) model are consolidated and constructed a generative model. With the given two inputs, the most abstract features generated through the layer-wise training of deep architecture and initialize the FFNN for fine-tuning. Consolidating unsupervised feature extraction as well as high-level feature representation and classification in one model makes the DBN stronger and enhance performance mutually in feature representation and classification.
1) Deep Belief Neural Network (DBN): In this section, a probabilistic generative model with deep architecture socalled Deep Belief Networks (DBN) [17] is presented. As shown in Fig. 3, the DBN is constructed by stacking predefined number of restricted Boltzmann Machines (RBM) (Section II-.2). It models the joint distribution between visible input $(v)$ and the $N$ hidden layers $h_{k}$ as follows:

$$
P\left(v, h^{1}, \ldots, h^{l}\right)=\left(\prod_{k=0}^{l-2} P\left(h^{k} \mid h^{k+1}\right)\right) P\left(h^{l-1}, h^{l}\right)
$$

where $v=h^{0}, p\left(h^{k-1} \mid h^{k}\right)$ is a conditional distribution for the visible units conditioned on the hidden units of the RBM at level $k$, and $P\left(h^{l-1}, h^{l}\right)$ is the visible-hidden joint distribution in the top-level RBM.

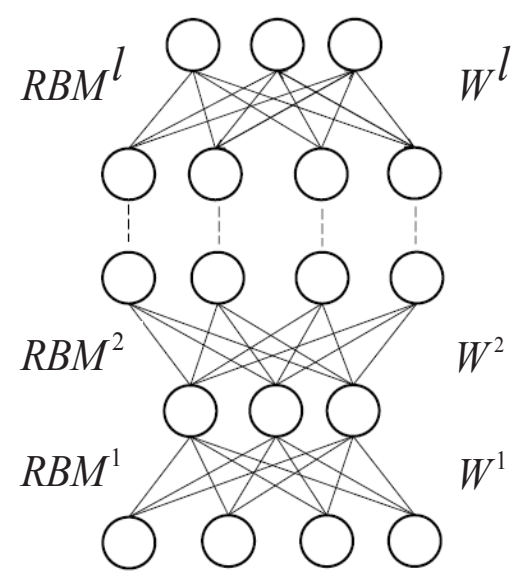

Fig. 3. Deep Belief Network (DBN) Architecture

In order to extract a deep hierarchical representation of the training (input) data, the DBN in Fig. 3 is trained in a greedy layer-wise manner. During the training, each RBMs are applied recursively with the features activations extracted by one RBM acting as the data for training the next RBM in the stack. After training stacked RBMs, the bottom up recognition weights of the resulting $\mathrm{DBN}$ is used for initialization of multi-layer feed-forward neural network (FFNN) which performs fine-tuning activity via backpropagation of error derivatives.

2) Restricted Boltzmann Machines (RBM): RBM [18] is a graphical model with observed data variables (visible neurons) and latent variables (hidden neurons). A typical RBM model topology is presented in Fig. 4 in which $m$ visible neuron $\left(v=\left(v_{1}, \ldots, v_{m}\right)\right)$ and $n$ hidden neurons $(h=$ $\left.\left(h_{1}, \ldots, h_{n}\right)\right)$ are fully connected via symmetric undirected weights. There is no intra-layer connections within either the visible or the hidden layer.

With the weights and biases, the energy of a joint configuration between hidden and visible neurons, $E(v, h)$ is defined as:

$$
E(v, h ; \theta)=-\sum_{i=1}^{n} \sum_{j=1}^{m} w_{i j} h_{i} v_{j}-\sum_{j=1}^{m} b_{j} v_{j}-\sum_{i=1}^{n} c_{i} h_{i}
$$




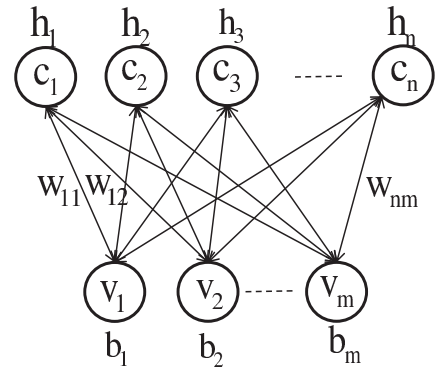

Fig. 4. A Restricted Boltzmann Machine (RBM)

where $w_{i j}$ is associated weight between $v_{j}$ and $h_{i}$ for all $i \in\{1, \ldots, n\}$ and $j \in\{1, \ldots, m\} ; b_{j}$ and $c_{i}$ are bias terms associated with the $j^{\text {th }}$ and $i^{t h}$ visible and hidden neurons; $\theta=\{W, b, c\}$ is model paper with symmetric weight parameters $W_{n \times m}$.

Through the energy function in (2), for all possible pair of visible $(v)$-hidden $(h)$ neurons, the networks assigns a joint probability distribution as follows:

$$
p(v, h)=\frac{e^{-E(v, h)}}{Z}
$$

where $Z=\sum_{v, h} e^{-E(v, h)}$ is the normalized term which is obtained by summing over the energy of all possible $(v, h)$ configurations.

Through the gradient of log probability of a visible vector $(v)$ over the weight $w_{i j}$, the updated rule is calculated by constructive divergence (CD) [19] algorithm as follows:

$$
\Delta w_{i j}=\eta\left(\left\langle v_{j} h_{i}\right\rangle_{\text {data }}-\left\langle v_{j} h_{i}\right\rangle_{\text {recon }}\right)
$$

where $\eta$ is the learning rate, (4), $\left\langle v_{j} h_{i}\right\rangle_{\text {recon }}$ is the reconstruction of original visible units which is calculated by setting the visible unit to random training vector. Due to the constraints in hidden to hidden connections and visible to visible connections, the binary state of hidden unit and visible unit are considered as follows:

$$
\begin{aligned}
& p\left(h_{i}=1 \mid v\right)=\sigma\left(\sum_{j=1}^{m} w_{i j} v_{j}+c_{i}\right) \\
& p\left(v_{j}=1 \mid h\right)=\sigma\left(\sum_{j=1}^{n} w_{i j} h_{i}+b_{j}\right)
\end{aligned}
$$

where $\sigma$ is the logistic sigmoid function $\sigma(x)=$ $1 /(1+\exp (-x))$.

\section{A. Performance Measurement}

To determine the performance of proposed detection system, Sensitivity and Specificity are introduced:

$$
\begin{aligned}
& \text { Sensitivity }=\frac{N_{T P}}{N_{T P}+N_{F N}} \\
& \text { Specificity }=\frac{N_{T N}}{N_{T N}+N_{F P}}
\end{aligned}
$$

where $N_{T P}$ is defined as number of true positive, $N_{F N}$ is number of false negative, $N_{F P}$ is number of false positive, and $N_{T N}$ is number of true negative. The values of these are within 0 to 1 . In this clinical study, the sensitivity is more important than the specificity because it mainly represents the performance of classifier. The higher sensitivity represents the better performance of the detection system.

\section{RESULTS AND DISCUSSION}

To study the natural occurrence of nocturnal hypoglycemia, 15 children with T1DM are monitored for 10hours overnight at the Princess Margaret Hospital for Children in Perth, Western Australia. The required physiological parameters are measured by the use of the non-invasive monitoring system, while the actual blood glucose levels (BGL) are collected as reference using Yellow Spring Instruments. The main parameters which are used for the detection of hypoglycemia are the heart rate (HR) and corrected QT (QTc). The response from the 15 children with T1DM [9] exhibit significant changes during their hypoglycemia phase in contrast to the non-hypoglycemia phase.

The overall data set consist of both hypoglycemia data part and non-hypoglycemia data part and organized into a training set, a validation set and a testing set which consists of randomly selected five patients data. To tackle the problem of T1DM, the hypoglycemia episodes (BGL $\leq 3.3 \mathrm{mmol} / l$ ) are detected by the use of DBN-based hypoglycemia monitoring system. The clinical results for hypoglycemia detection with different methods are tabulated in Table I and II. For comparison studies, deep belief network (DBN) block based neural network (BBNN) [10], wavelet neural network (WNN) [10], feedforward neural network (FFNN) [10], and multiple regression (MR) models are analyzed.

TABLE I

PERformance Comparisons: MEAN Training, VALidation and TESTING

\begin{tabular}{|l|c|c|l|l|l|l|}
\hline & & DBN & BBNN & WNN & FWNN & MR \\
\hline \multirow{3}{*}{ Training(\%) } & Sensitivity & 92.31 & 84.85 & 84.12 & 83.64 & 81.82 \\
& Specificity & 40.00 & 40.35 & 40.63 & 40.50 & 41.32 \\
& Gamma & 71.38 & 68.16 & 66.72 & 66.38 & 65.62 \\
\hline \multirow{3}{*}{ Validation(\%) } & Sensitivity & 92.80 & 87.34 & 80.44 & 79.07 & 82.19 \\
& Specificity & 42.86 & 40.13 & 40.94 & 41.38 & 40.75 \\
& Gamma & 72.84 & 68.46 & 64.64 & 63.94 & 65.07 \\
\hline \multirow{3}{*}{ Testing(\%) } & Sensitivity & 79.70 & 76.28 & 71.39 & 68.34 & 64.65 \\
& Specificity & 50.00 & 50.40 & 44.37 & 48.34 & 53.09 \\
& Gamma & 67.82 & 65.93 & 60.58 & 60.64 & 60.03 \\
\hline
\end{tabular}

As can be seen in Table I, the average (mean) testing result of proposed DBN-based hypoglycemia system found to be satisfactory by giving the sensitivity and specificity of (79.70\% and 50\%). Besides, in Table II, the performance of DBN is better than other classifiers by achieving the best testing sensitivity, $80.00 \%$ and acceptable specificity $50.00 \%$. In this study, $\gamma$ analysis is defined as $\gamma=\theta \xi+(1-\theta) \eta,(\theta \in$ $[0,1])$ for evaluation of the proposed system performance. 
TABLE II

BEST TESTING RESULTS COMPARISONS

\begin{tabular}{|l|l|l|l|}
\hline Method & Sensitivity & Specificity & Gamma \\
\hline \hline DBN & $\underline{\mathbf{8 0 . 0 0}}$ & $\underline{\mathbf{5 0 . 0 0}}$ & $\underline{\mathbf{6 8 . 0 0}}$ \\
BBNN [10] & 76.74 & 52.73 & 67.14 \\
FRM [9] & 75.00 & 50.00 & 65.56 \\
WNN [10] & 72.09 & 45.45 & 61.43 \\
FWNN [10] & 69.77 & 49.09 & 61.50 \\
MR & 65.12 & 57.27 & 61.98 \\
\hline
\end{tabular}

Since the minimum requirement of a hypoglycemia detection system is $60 \%$ of sensitivity and $40 \%$ of specificity, $\theta$ is set to 0.6 in this analysis. As can be seen in Table I and II, in terms of $\gamma$ analysis, the proposed DBN outperforms other classifiers with $\gamma$ value of $67.82 \%$ (mean) and $68.00 \%$ (best). In short, the DBN is a superior classifier in detection of hypoglycemia episodes.

To develop DBN-based hypoglycemia monitoring system (Fig. 2), the DBN is formed by training the individual RBM layer one over another. During the training, the hidden layer RBM is randomly initialized and updated using (4) with learning rate $(\eta)$ of 0.1 until 100 epoches. In this study, a single layer RBM with 20 hidden neurons is good enough, i.e., the optimal structure of DBN is [2-20-1]. It means that there are 2 visible units at the input layer, 20 hidden unit at RBM layer and 1 output units at final classification layer.

Since the architecture of DBN, especially the size of DBN can greatly influence its performance, it is vital to carefully select the number of hidden RBM units and/or layers. There is trade-off between too many and too few number of hidden neurons and/or layers. To the best of knowledge, there is no special techniques proposed for the selection of optimal DBN parameters. In general, all optimal parameters are selected by the use of trial-error analysis.

\section{CONCLUSIONS}

For detection of the hypoglycemic episodes for diabetes patients an intelligent diagnostic system using deep learning framework, namely deep belief network has been developed. The experimental results indicate that hypoglycemic episodes in T1DM children can be detected non-invasively and effectively by the use of real time physiological parameters of ECG signal. The performance evaluation is done in comparison with other classifiers (DBN, BBNN, WNN, FWNN, and MR). The improvement in sensitivity and specificity is satisfactorily found at $80.00 \%$ and $50.00 \%$. In short, the proposed intelligent system can detect episodes of hypoglycemia effectively and efficiently. There is one limitation for the proposed hypoglycemia detection. All the data are recorded from children with T1DM and there are no heart problems to affect the heart rate and QT interval. As a consequence, there are no confounding conditions included in these data sets. However, the detection performance may be affected if the patients have heart problems.

\section{REFERENCES}

[1] J. C. Pickup, "Sensitivity glucose sensing in diabetes", Lancet, vol.355, no. 1 , pp. $426-427,2000$.

[2] DCCT Research Group, "Adverse events and their association with treatment regimens in the Diabetes Control and Complications Trial", Diabetes Care, vol.18, pp. 1415-1427, 1995.

[3] DCCT Research Group, "Epidemiology of severe hypoglycemia in the diabetes control and complication trial", The American Journal of Medicine, vol.90, no. 4, pp. 450-459, 1991.

[4] C. E. F. Amaral and B. Wolf, "Current development in non-invasive glucose monitoring", Medical Engineering and Physics, vol.30, no. 5, pp. 541-549, 2008

[5] Directnet Study Group, "Evaluation of factors affecting CGMS calibration", Diabetes Technology and Therapeutics, vol.8, pp. 318-325, 2006.

[6] N. D. Harris, S. B. Baykouchev and J. L. B. Marques, "A portable system for monitoring physiological responses to hypoglycaemia", Journal of Medical Engineering and Technology, vol.20, no.6, pp. 196202, 1996.

[7] J. L. B. Marques1 and E. George, "Altered ventricular repolarization during hypoglycaemia in patients with diabetes", Diabetic Medicine, vol.14, no.8, pp. 648654, 1997.

[8] P.P San, S.H. Ling, and H.T. Nguyen, Block based neural network for hypoglycaemic detection, in Proc. 33nd Annual International conference of the IEEE Engineering in Medicine and Biology Society, Boston, USA, Aug 2011, pp. 5666-5669.

[9] S. H. Ling, Nuryani and H. T. Nguyen, "Evolved Fuzzy Reasoning Model for Hypoglycemic Detection", in Proc. 32nd Annual International conference of the IEEE Engineering in Medicine and Biology Society, Argentina, Aug 2010, pp 4662-4665.

[10] P. P. San, S. H. Ling, Nuryani, H. T. Nguyen, "Evolvable RoughBlock-Based Neural Network and its Biomedical Application to Hypoglycemia Detection System", in Proc. 32nd Annual International conference of the IEEE Engineering in Medicine and Biology Society, vol. 44, no. 8, pp. 1338-1349, 2013.

[11] G. E. Hinton, "Learning multiple layers of representation", Trends in Cognitive Sciences, vol. 11, no. 10, pp. 428-434, 2007.

[12] G. E. Dahl, M. Ranzato, A. Momamed and G. E. Hinton, "Phone recognition with the mean-covariance restricted noltzmann machines", Advances in Neural Information Processing Systems of NIPS, 2010.

[13] H. P. Martinez, Y. Bengio and G. N. Yannakakis, "Learning multiple layers of representation", IEEE Computational Intelligence Magazine, vol. 8, no. 2, pp. 20-33, 2013.

[14] V. Srinivasan, C. Eswaran and N. Sriraam, "Approximate EntropyBased Epileptic EEG Detection Using Artificial Neural Networks", IEEE Transactions on Information Technology in Biomedicine, vol. 11, no. 3, pp. 288-295, 2007.

[15] G. Heger, K. Howorka and H. Thoma, "Monitoring set-up for selection of parameters for detection of hypoglycaemia in diabetic patients", Diabetologia, vol.34, pp. 69-75, 1996.

[16] H. T. Nguyen, N. Ghevondian and T. W. Jones, "Detection of nocturnal hypoglycemic episodes (natural occurrence) in children with Type 1 diabetes using an optimal Bayesian neural network algorithm", 30th Annual International Conference of the IEEE Engineering in Medicine and Biology Society, pp. 1311-1314, 2008.

[17] G. E. Hinton, S. Osindero and Y. W. Teh, "A fast learning algorithm for deep belief nets", Neural Computation, vol. 18, no. 7, pp. 15271554,2006

[18] A. Fischer and C. Igel, "An introduction to rstricted boltzmann machines", Progress in Pattern Recognition, Image Analysis, Computer Vision, and Applications, vol. 7441, pp. 14-36, 2012.

[19] R. Sarikaya, G. E. Hinton and A. Deoras, "Application of deep belief networks for natural language understanding", IEEE/ACM Transactions on Audio, Speech and Language Processing, no. 22, vol. 4, pp. 778-784, 2014. 